\title{
Negative Knight Shift in Ba-Ti Oxyhydride: An Indication of the Multiple Hydrogen Occupation
}

Tai Misaki, Itaru Oikawa, Hitoshi Takamura

\begin{abstract}
Department of Materials Science, Graduate School of Engineering, Tohoku University, Sendai 980-8579, Japan
\end{abstract}

\section{Structural characterization of synthesized Ba-Ti oxyhydrides}

Incorporation of hydrogen into $\mathrm{BaTiO}_{3}$ caused change in the crystal structure from tetragonal to cubic as indicated in the (200) diffraction peak in Fig. S1 (a). The diffraction peak of (200) split into (200) and (002) peaks due to the difference in the length of a- and c-axis of tetragonal $\mathrm{BaTiO}_{3}$. The (002) diffraction peak disappeared and only a single peak of (200) diffraction is observed in the Ba-Ti oxyhydrides which indicates the transition to cubic symmetry due to the hydrogen incorporation. This transition decreases the cell volume. Fig. $\mathrm{S} 1$ (b) shows cell volume of $\mathrm{BaTiO}_{3}$ and $\mathrm{Ba}-\mathrm{Ti}$ oxyhydrides. The lattice contraction compared to $\mathrm{BaTiO}_{3}$ in $\mathrm{Ba}-\mathrm{Ti}$ oxyhydride reduced for $12 \mathrm{~h}$ is originated from the symmetry change. Once hydrogen is incorporated into the lattice and the symmetry is changed, the cell volume turns to increase due to the reduction expansion. When the reduction of the sample due to hydrogen incorporation occurs, conduction band is filled with electrons from hydrogen atoms and oxidation state of $\mathrm{Ti}^{4+}$ is reduced to $\mathrm{Ti}^{3+}$. This reduction increases the ionic radius of $\mathrm{Ti}$ and causes the lattice expansion.

(a)

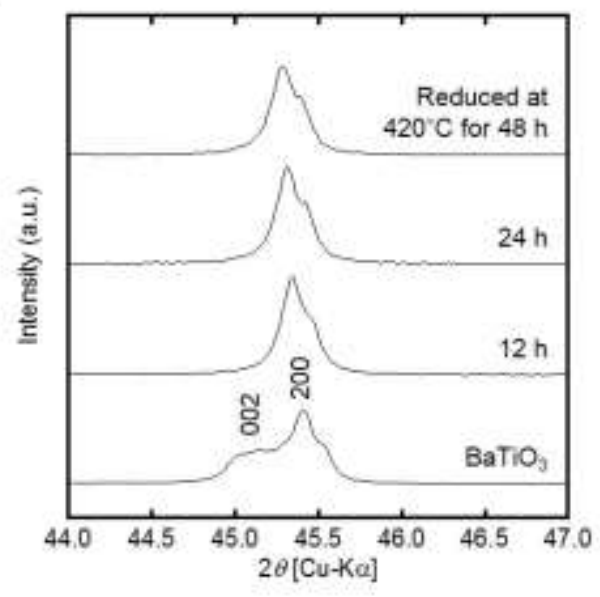

(b)

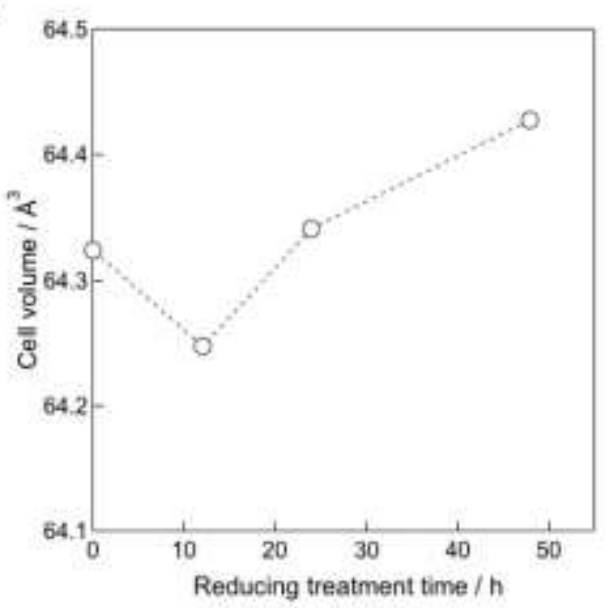


Figure S1 (a) Enlarged view of (200) diffraction peak in Fig. 1 and (b) Cell volume of Ba-Ti oxyhydride reduced at $420^{\circ} \mathrm{C}$ for different time.
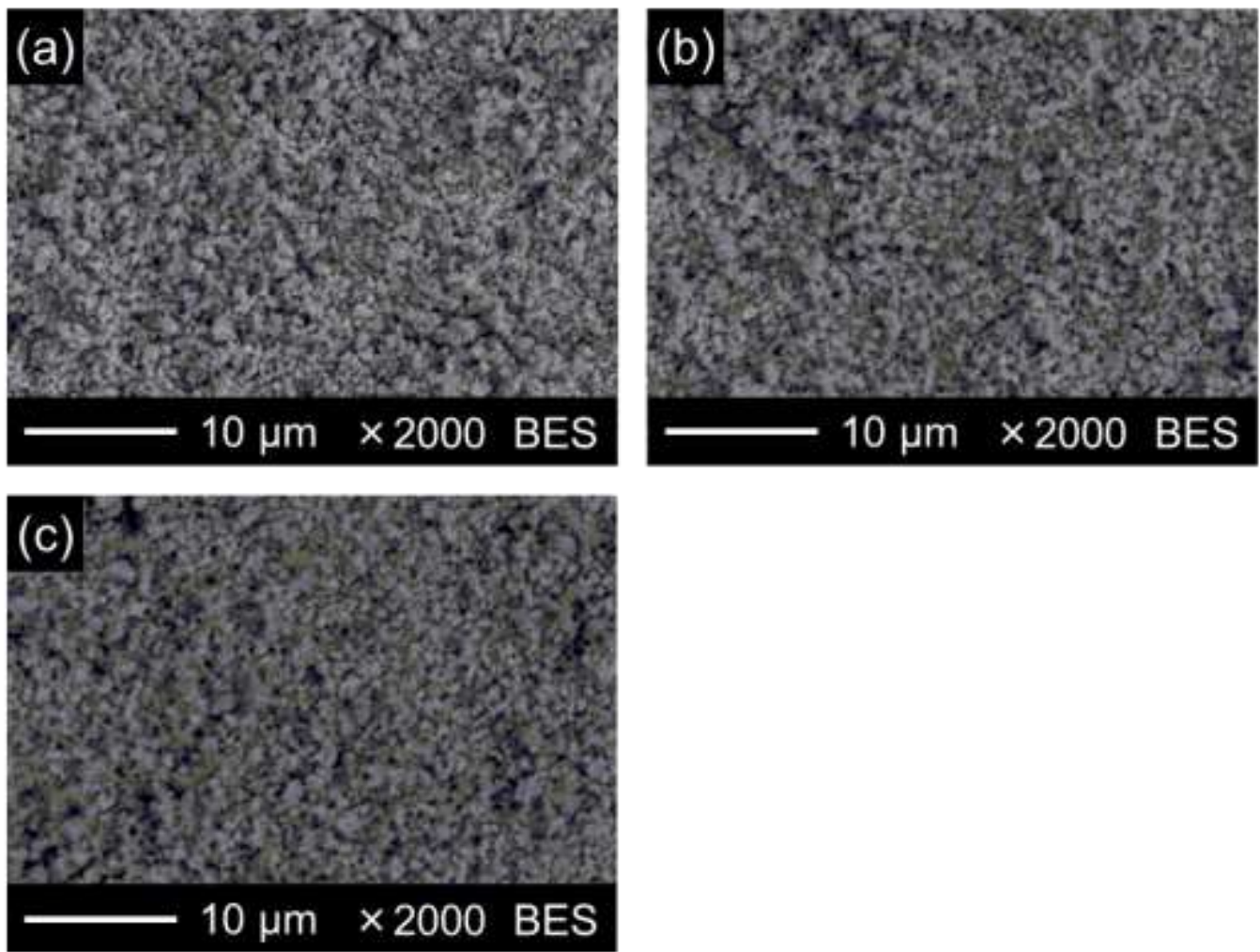

Figure S2 SEM images of Ba-Ti oxyhydrides reduced at $420^{\circ} \mathrm{C}$ for 12,24 and $48 \mathrm{~h}$.

\section{Evaluation of hydrogen amounts by inert gas fusion technique}

The amounts of hydrogen in the sample were evaluated by the inert gas fusion technique. The sample was put into a $\mathrm{Sn}$ capsule and fused in a carbon crucible. The exhaust hydrogen gas was oxidized to $\mathrm{H}_{2} \mathrm{O}$ by flowing through copper oxides and detected by infrared absorption spectroscopy. Fig. S3 (a) shows the gas extraction curve for the samples reduced at $420^{\circ} \mathrm{C}$ for 12,24 and $48 \mathrm{~h}$. The peak area of the extraction curve was converted to hydrogen concentration by using the calibration curve obtained from the hydrogen concentration in $\mathrm{CaH}_{2}$. The hydrogen extraction curves of $\mathrm{CaH}_{2}$ in different weight was measured and the calibration curve between the peak area of the extraction curve and the hydrogen concentration were obtained by averaging the results of five samples. Fig. S3 (b) shows the hydrogen concentration of the sample with different reducing times. The hydrogen concentration of each sample was obtained from the average value of three samples. 
(a)
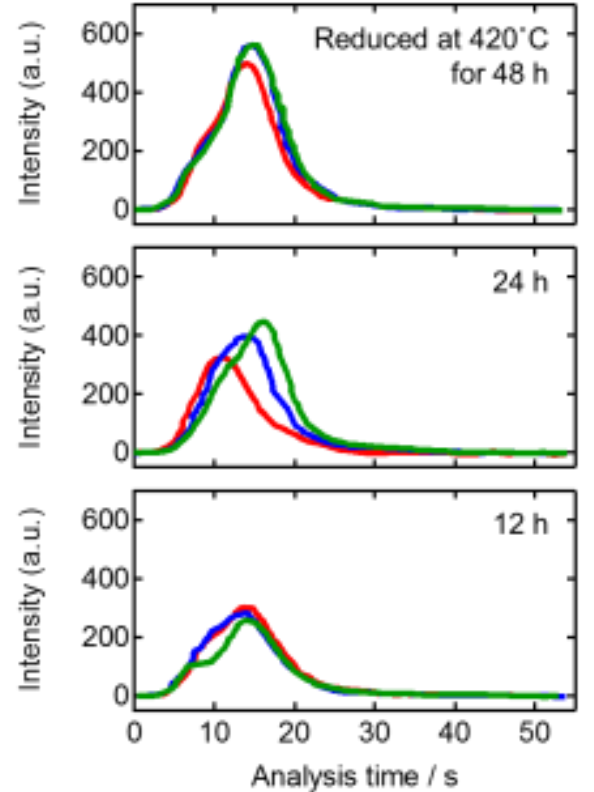

(b)

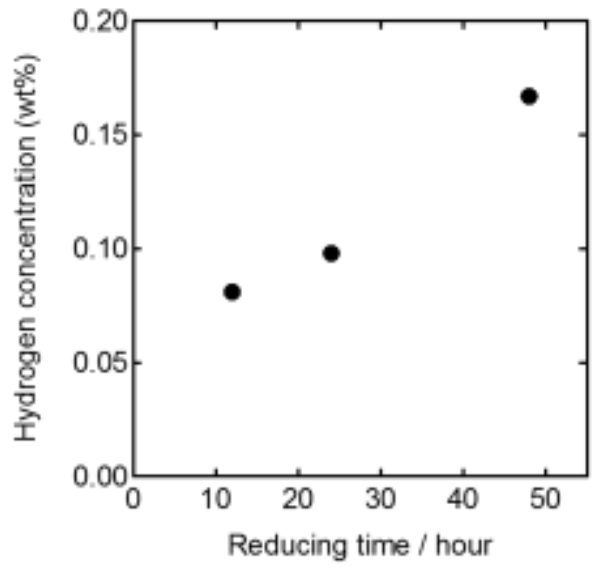

Figure S3 (a) Hydrogen gas extraction curves for different reducing times and (b) average hydrogen concentration determined from the peak area of the extraction curves.

Peak area, chemical shift and full width at half maxima of peak 1 and peak 2 in ${ }^{1} \mathrm{H}$ MAS NMR spectra of Ba-Ti oxyhydrides

(a) Peak 1

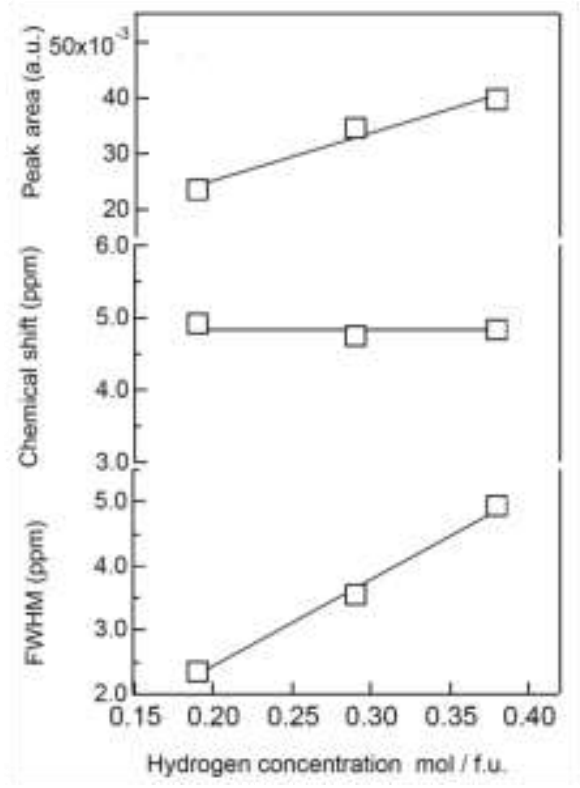

(b) Peak 2

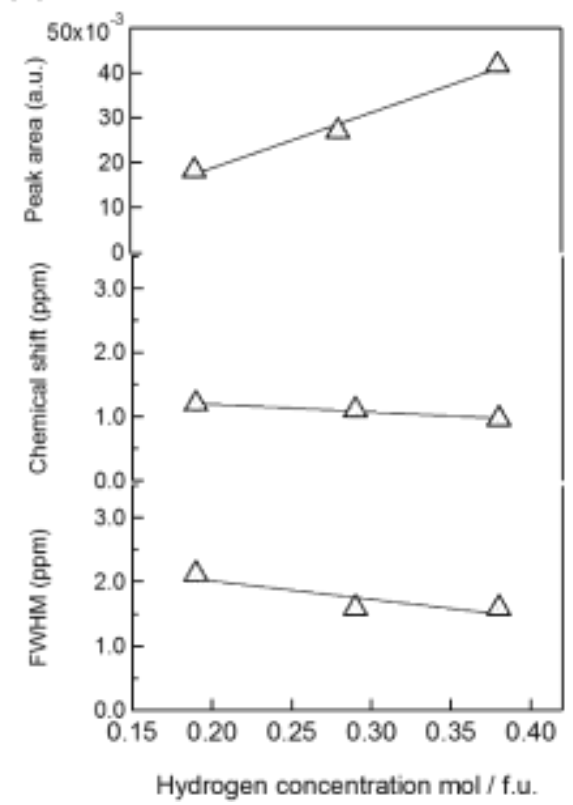

Figure S4 Peak area, chemical shift and full width at half maxima (fwhm) of (a) peak 1 and (b) peak 2 in ${ }^{1} \mathrm{H}$ MAS NMR spectra of the Ba-Ti oxyhydride at different hydrogen concentrations 


\section{Influence of impurities investigated by high temperature XRD}

High temperature XRD measurements are carried out to investigate Ca-containing impurities and other impurities which are difficult to observe by room temperature XRD due to their low crystallinity or the formation of amorphous phases. Ca-containing impurities can originate from the resultant $\mathrm{Ca}$ compounds and the unreacted $\mathrm{CaH}_{2}$ in the topochemical reaction. Even though these compounds are removed from the sample by washing with $\mathrm{NH}_{4} \mathrm{Cl} / \mathrm{methanol}$ solution and distilled water, it is likely that a small quantity remains in the sample. It is assumed that the impurities are $\mathrm{Ca}(\mathrm{OH})_{2}$ because the residual $\mathrm{Ca}$ compounds of the topochemical reaction are likely to be $\mathrm{CaO}$ and $\mathrm{CaH}_{2}$, and these residuals are reacted with air and water to form $\mathrm{Ca}(\mathrm{OH})_{2}$ during the washing process.

XRD patterns of the Ca-containing impurities are collected by increasing the temperature from 100 to $800^{\circ} \mathrm{C}$. High temperature XRD measurements were carried out at D8 ADVANCE (Bruker) with an HTK 1200N high temperature oven chamber (Anton Paar) and samples were placed on an $\mathrm{Al}_{2} \mathrm{O}_{3}$ sample holder. The measurements were carried out in an $\mathrm{Ar}$ atmosphere with $2 \theta$ ranging from $28-58^{\circ}$. If there are detectable amounts of $\mathrm{CaO}$ and $\mathrm{Ca}(\mathrm{OH})_{2}$ in the sample as impurity phases which cannot be detected due to their low crystallinity or the formation of amorphous phases, it should be possible to observe their crystallization or the decomposition of $\mathrm{Ca}(\mathrm{OH})_{2}$ into $\mathrm{CaO}$ as the temperature increases in the measured atmosphere. ${ }^{1}$ Fig. S5 shows the temperature dependence of the XRD patterns of the Ba-Ti oxyhydride reduced at $420^{\circ} \mathrm{C}$ for $48 \mathrm{~h}$. The near baseline region of the XRD patterns is enlarged to identify the minor phases. No detectable peaks related to $\mathrm{CaO}$ and $\mathrm{Ca}(\mathrm{OH})_{2}$ are observed in at any of the measured temperatures: this confirms the amounts of $\mathrm{CaO}$ and $\mathrm{Ca}(\mathrm{OH})_{2}$ are below the detection limit of the present measurement. In addition, the XRD patterns show no indication of the crystallization behavior of additional phases up to $800^{\circ} \mathrm{C}$ in the measured $2 \theta$ range. This result implies the amounts of amorphous impurities which may contain hydrogens are also below the detection limit. 


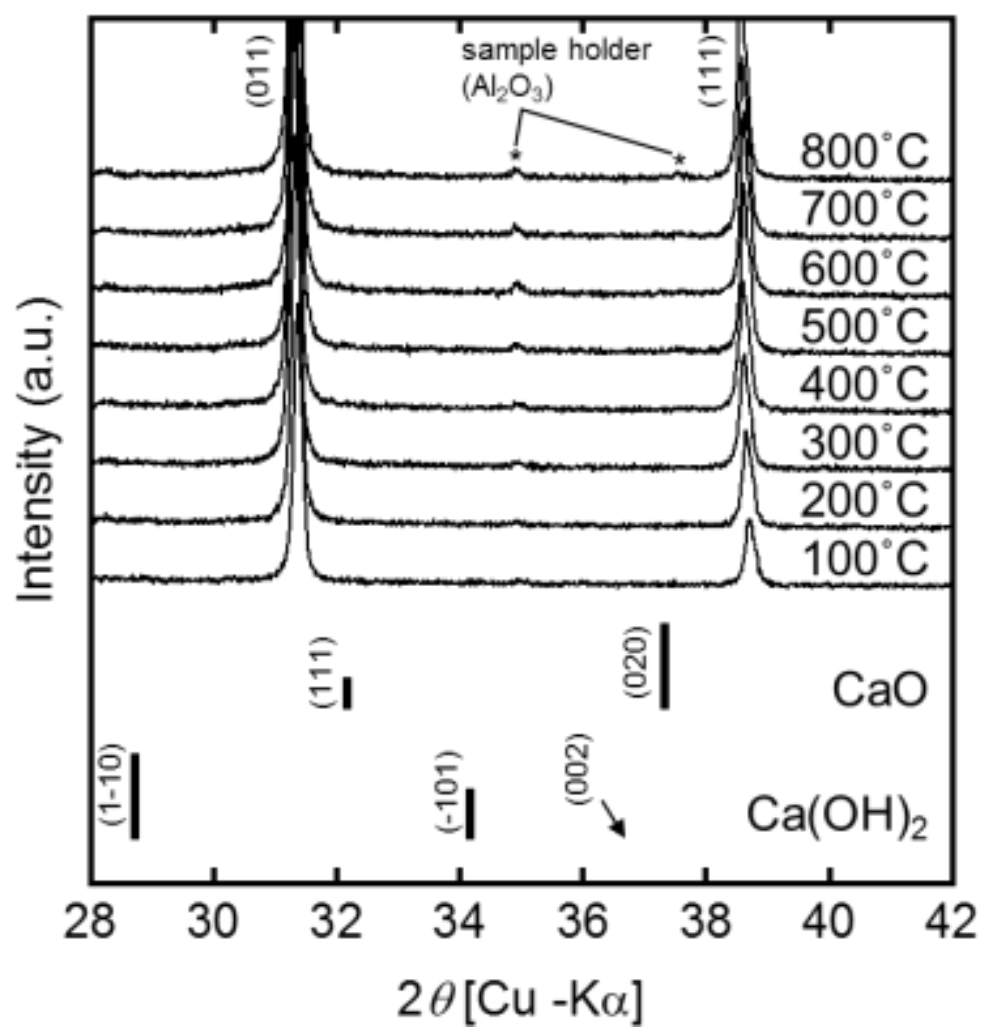

Figure S5 Temperature dependence of XRD patterns of the Ba-Ti oxyhydride reduced at $420^{\circ} \mathrm{C}$ for $48 \mathrm{~h}$. The diffraction patterns of $\mathrm{CaO}$ and $\mathrm{Ca}(\mathrm{OH})_{2}$ are indicated as a reference.

\section{Spin-lattice relaxation rate of Ba-Ti oxyhydrides}

Assuming that the core-polarization interaction dominates the hyperfine interaction of hydrogen atoms in Ba-Ti oxyhydrides, and the contribution from the Fermi contact shift and the orbital motion of $d$ electrons can be neglected, the spin-lattice relaxation rate, $T_{1 \mathrm{e}^{-1}}$ derived from the hyperfine interaction with conduction electrons is express as follows, ${ }^{2}$

$$
\frac{1}{T_{1 e} T}=4 \pi \hbar \gamma_{I}^{2} k_{B}\left[H_{h f}(d) N_{d}\left(E_{F}\right)\right]^{2} q
$$

where $\hbar$ is Plank constant, $\gamma_{1}$ is the gyromagnetic ratio of nucleus $I, k_{\mathrm{B}}$ is Boltzmann constant, $H_{\mathrm{hf}}(d)$ is the hyperfine field due to the core polarization interaction caused by the unpaired $d$ electrons, $N_{d}\left(E_{\mathrm{F}}\right)$ is the $d$-band density of states at the Fermi level, $q$ is the reduction factors resulting from $d$-electron orbital degeneracy at $E_{\mathrm{F}}{ }^{3}$ According to the equation above, when the core polarization interaction dominates the spin-lattice relaxation of ${ }^{1} \mathrm{H}$, the square root of the relaxation rate, $T_{1 \mathrm{e}}{ }^{-1 / 2}$, is proportional to the density of $d$ electrons at $E_{\mathrm{F}}$. Fig. S6 shows $T_{1 \mathrm{e}}{ }^{-1 / 2}$ with respect to the hydrogen 
concentration of the Ba-Ti oxyhydride samples. The value of $T_{1 \mathrm{e}^{-1 / 2}}$ increases with increasing hydrogen concentration in the sample which indicates conduction electrons increase as hydrogen concentration increases and ${ }^{1} \mathrm{H}$ is interact with conduction band electrons via core polarization interaction. However, it is difficult to conclude that the core polarization interaction dominates the spin-lattice relaxation of ${ }^{1} \mathrm{H}$ because data points are insufficient to attribute the $T_{1 \mathrm{e}^{-1 / 2}}$ trend to the core polarization interaction.

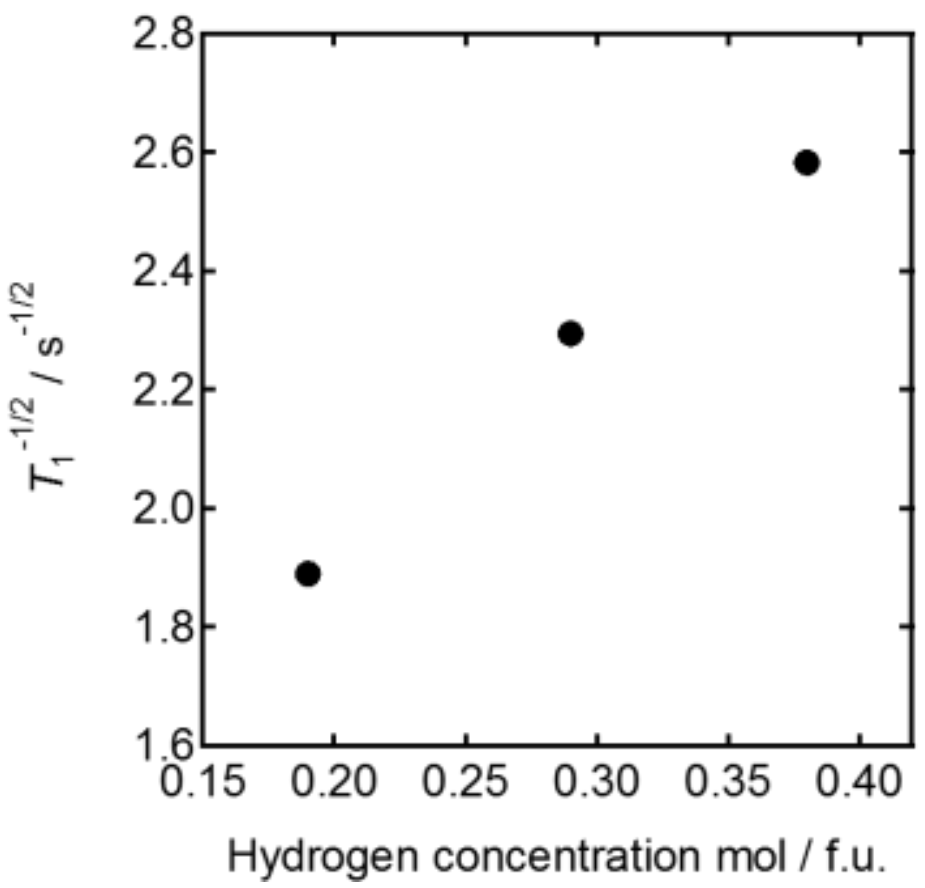

Figure S6 Hydrogen concentration dependence of the square root of the spin-lattice relaxation rate of peak 3 in ${ }^{1} \mathrm{H}$ MAS NMR spectra of Ba-Ti oxyhydrides.

\section{Synthesis and characterization of Ba-Ti oxydeuteride}

Prior to the synthesis of Ba-Ti oxydeuterides, a successful synthesis of calcium deuteride was confirmed by a powder XRD pattern and Raman spectra shown. A XRD pattern of synthesized $\mathrm{CaD}_{2}$ is shown in Fig. $\mathrm{S} 7$ (a). A single phase of $\mathrm{CaD}_{2}$ with space group of Pnma, as reported in the previous study, ${ }^{4}$ is confirmed. Fig. S7 (b) shows Raman spectra of synthesized $\mathrm{CaD}_{2}$ along with the spectrum of $\mathrm{CaH}_{2}$. Based on a previous report, Raman peaks typical for $\mathrm{CaH}_{2}$ related to vibrational motions of hydride ions are observed at 1000, 747 and $649 \mathrm{~cm}^{-1} .^{5}$ In the Raman spectra of $\mathrm{CaD}_{2}$, a shift in these three peaks to higher wave number is observed which indicates isotope effects between hydrogen and deuterium. 

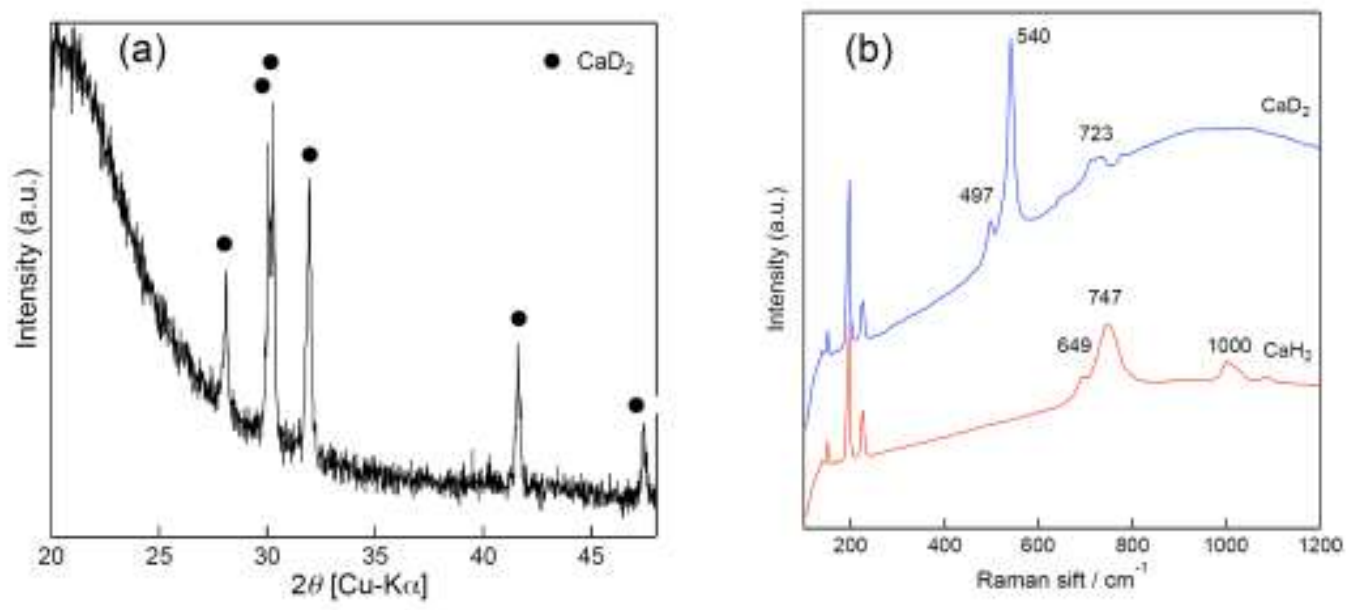

Figure S7 (a) powder XRD pattern of calcium deuteride and (b) Raman spectra of calcium deuteride

Ba-oxydeuterides were successfully synthesized using $\mathrm{CaD}_{2}$. From the XRD pattern of Ba-Ti oxydeuteride synthesized at $420^{\circ} \mathrm{C}$ for $48 \mathrm{~h}$ in reducing atmosphere shown in Fig. S8 (a), a single phase of perovskite-type structure is confirmed. The local structure of ${ }^{1} \mathrm{H}$ in the oxydeuteride is analyzed by ${ }^{1} \mathrm{H}$ NMR. Fig. S8 (b) shows ${ }^{1} \mathrm{H}$ magic-angle spinning (MAS) NMR spectrum of the Ba-Ti oxydeuteride. Since $\mathrm{CaD}_{2}$ is used for synthesis of Ba-Ti oxydeuteride, ${ }^{1} \mathrm{H}$ signals in Fig. S8 (b) is originated not from the products of the topochemical reaction but from hydrogen-containing species incorporated after the synthesis such as $\mathrm{Ca}(\mathrm{OH})_{2}$ due to hydroxidization of $\mathrm{CaD}_{2}$ and adsorbed water. Two peaks are observed in the ${ }^{1} \mathrm{H}$ spectra of the Ba-Ti oxydeuteride. A peak at $1.0 \mathrm{ppm}$ can be assigned to ${ }^{1} \mathrm{H}$ in $\mathrm{Ca}(\mathrm{OH})_{2}$ according to the chemical shift. Another peak at $5.1 \mathrm{ppm}$ is presumably originated from adsorbed water. 

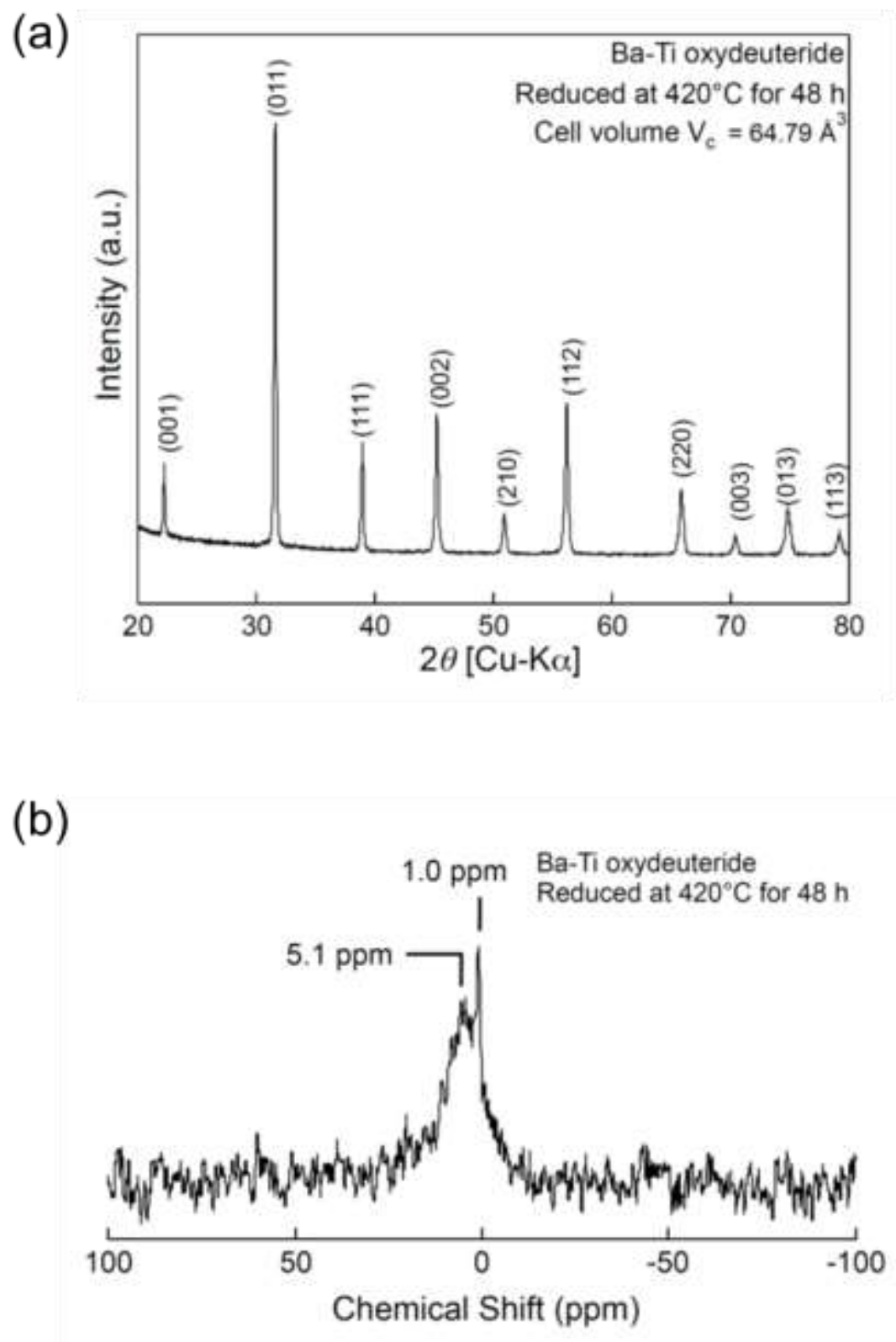

Figure S8 (a) XRD pattern of Ba-Ti oxydeuteride. (b) ${ }^{1} \mathrm{H}$ MAS NMR spectrum of Ba-Ti oxydeuteride.

\section{References}

1. Halstead, P. E.; Moore, A. E. The thermal dissociation of calcium hydroxide. J. Chem. Soc. 1957, 3873-3875.

2. Bowman, R. C. Jr.; Venturini, E. L.; Craft, B. D.; Attalla, A.; Sullenger, D. B. Electronic structure of zirconium hydride: A proton NMR study. Phys. Rev. B 1983, 27, 
1474-1488.

3. Narath, A. in Hyperfine Interactions, ed. Freeman, A. J.; Frankel, R. B. Academic, New York, 1967, 287.

4. Andresen, A. F.; Maeland, A. J.; Slotfeldt-Ellingsen, D. Calcium hydride and deuteride studied by neutron diffraction and NMR. J. Solid State Chem. 1977, 20, 93-101.

5. Li, B.; Li, Y.; Yang, K.; Cui, Q.; Ma, Y.; Zou, G. Raman evidence of a new high-pressure phase in calcium hydride. J. Phys.: Condens. Matter 2007, 19, 226205. 